\title{
Construcción y Validación de un Instrumento para Evaluar las Características de la Responsabilidad Social Universitaria en Estudiantes Universitarios
}

\author{
Construction and Validation of an Instrument to Evaluate the \\ Characteristics of University Social Responsibility in University \\ Students
}

\author{
Valentina Bolio Domínguez ${ }^{1 *}$ \\ Leny Michele Pinzón Lizarraga ${ }^{2}$ \\ ${ }^{1}$ Universidad Marista de Mérida, México \\ ${ }^{2}$ Instituto Tecnológico de Mérida, México
}

\begin{abstract}
En este artículo se presentan los resultados obtenidos del diseño, construcción y validación estadística de un instrumento para la autoevaluación de las características vinculadas de los estudiantes a la Responsabilidad Social Universitaria (RSU). Aunque se tienen propuestas teóricas diferentes sobre RSU, apenas existe un desarrollo incipiente en la construcción de instrumentos de evaluación que permitan a las Universidades reflexionar si sus esfuerzos en la formación de estudiantes socialmente responsables dan como resultados profesionales con las características necesarias para incidir en la transformación social. La construcción de un instrumento que conjuntara las características de RSU se hizo en cinco etapas: el trabajo de diseño y construcción estuvo conformado por tres, mientras que el de pilotaje, validación e instrumento final por dos. La revisión bibliográfica permitió crear una escala final de 47 reactivos agrupados en siete características de un alumno socialmente responsable: conciencia, compromiso, controversia con civilidad, respeto a la diversidad, ciudadanía, justicia social y cambio. Asimismo, los reactivos se distribuyen en cuatro dimensiones que responden al Modelo de Cambio Social de Retolaza (2010), a saber: transformación personal, transformación de relaciones, transformación de patrones culturales y transformación estructural. El instrumento fue sometido a la validación de contenido a través de ocho jueces expertos y, posteriormente, aplicado a una muestra piloto de 102 estudiantes de dos universidades privadas. El análisis estadístico arrojó un alto índice de consistencia interna en las 7 características y en cada una de las cuatro dimensiones. El análisis factorial mostró consistencia con el Modelo de Retolaza.
\end{abstract}

Descriptores: Responsabilidad social; Instrumento de medición; Autoevaluación; Cambio social; Transformación social.

The present article show results obtained from the construction and statistical validation of an instrument designed for the analysis of University Social Responsibility (RSU) characteristics in university students. Despite of the different theoretical proposals available about RSU, the development of measuring instrument related to social responsibility and their influence among students for social change is on its early stages of development. The construction of the instrument including different RSU characteristics was done in five stages: three were related to designing and construction and two were related to the application, validation, and development of the final version. Literature review allowed the creation of a scale of

*Contacto: vbolio@marista.edu.mx

Recibido: $\quad 2$ de febrero de 2019

ISSN: 2254-3139

$1{ }^{\text {a }}$ Evaluación: 26 de abril de 2019

ISSN: 2254-3139

www.rinace.net/riejs/

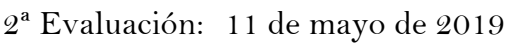

revistas.uam.es/riejs

Aceptado: 16 de mayo de 2019 
47 items divided into seven characteristics of a socially responsible student: consciousness, commitment, controversy with civility, respect to diversity, citizenship, social justice and change. The previous items can be grouped in four dimensions corresponding to Retolaza's Model of Social Change: personal transformation, transformation of relationships, transformation of cultural patterns, and structural transformation. The instrument was analyzed by eight experts in order to assess its content and validity followed by its evaluation in a pilot test with a sample including 102 students from two private universities. The statistical analysis showed high internal consistency in all seven characteristics and in each four dimensions. The factorial analysis showed consistency in all four dimensions found in the Retolaza model. The content validity of the instrument was analyzed by eight expert judges and subsequently the instrument was applied to a pilot sample of 102 students from two private universities. The statistical analysis showed a high general internal consistency and also high internal consistency in each of the dimensions. The factor analysis showed consistency in all four dimensions found in the Retolaza Model.

Keywords: Social responsibility; Measuring instrument; Self-evaluation; Social change; Changing society.

\section{Introducción}

Transformar la sociedad hacia formas más justas y pensar en un país que haga esfuerzos sistemáticos para poner fin a la pobreza, a las desigualdades y contrarrestar el cambio climático, son objetivos que hoy en día les competen a las Instituciones de Educación Superior (IES), inspirados por la agenda 2030 y los 17 Objetivos de Desarrollo Sostenible (ONU, 2019).

Los jóvenes que transitan por las Universidades son la principal fuerza impulsora que tienen las IES para encontrar caminos hacia la transformación social. En palabras de la UNESCO (2016, p. 24), "los jóvenes no son los ciudadanos del futuro sino los ciudadanos activos de ahora”. De ahí la necesidad de encontrar mecanismos institucionales para brindarles oportunidades sistemáticas que los formen como agentes impulsores del cambio social.

Uno de los mecanismos que las universidades han adoptado para formar ciudadanos y profesionales socialmente responsables se asocia con el desarrollo de acciones vinculadas al concepto de Responsabilidad Social Universitaria (RSU), el cual se define en palabras de Vallaeys (2018) como:

\section{La responsabilidad de la universidad por sus impactos en todos sus ámbitos de gestión, formación, cognición y participación social, dirigida hacia el desarrollo sostenible de la sociedad, y convocando transversalmente a toda la comunidad universitaria para implementar políticas y programas que transformen a la institución desde un autodiagnóstico reflexivo permanente de sus logros y fallas, con coherencia, transparencia y ánimo de innovación. (p. 55)}

De acuerdo a esta definición, una gestión universitaria responsable implicaría el análisis de los impactos, tanto positivos como negativos, los cuales deben administrarse desde un ejercicio ético y transparente teniendo como fin último la transformación social.

El impacto educativo, según Vallaeys (2016), es el que se relaciona con el cuestionamiento del tipo de profesional y ciudadano que se está formando. El análisis de este impacto abarca el conocimiento de las formas en las que el estudiante concibe al mundo y se comporta en él, y que tienen que ver con una escala de valores producto de su formación universitaria. Considerar este impacto como universidad implicaría no solo implementar acciones 
socialmente responsables, sino también la búsqueda o diseño de mecanismos que permitan evaluar si la Universidad está formando a profesionales socialmente responsables.

El diseño y administración de instrumentos de evaluación son mecanismos que permiten a las universidades conocer si están formando para la transformación social. El presente artículo presenta una propuesta de instrumento de autoevaluación que le posibilita a los estudiantes reconocer en su persona si poseen o no las características vinculadas a la RSU. La construcción de este instrumento forma parte de una tesis doctoral la cual lleva por nombre "Características vinculadas a la RSU presentes en estudiantes al momento de egreso y experiencias universitarias que favorecen su adquisición".

\section{Planteamiento del problema}

En el marco de la agenda 2030 y los ODS, la pobreza, desigualdad y deterioro del medio ambiente, son algunos de los problemas que representan hoy en día un desafío para las universidades. De continuar sin resolverse, ponen en entredicho la pertinencia social de las mismas, entendida como la capacidad de formar estudiantes que den respuesta a las problemáticas sociales de su entorno en apego a su misión, generando beneficios en los sectores más desfavorecidos (Tünnermann, 2013). Ante este panorama, ¿están las universidades formando para estar a la altura de estos desafíos? A continuación, se presentan algunas opiniones al respecto:

Cruz (2011, parr.1) señala que "nunca como hasta ahora parece haber existido tanta distancia entre lo que se espera que las Instituciones de Educación Superior asuman como parte de su responsabilidad con la sociedad y el modo en cómo éstas están respondiendo a esas expectativas". Por su parte, Vallaeys, (s.f., p.4) refiere que:

Es verdad que todos los líderes que hoy gobiernan las instituciones públicas y privadas
que dirigen este Titanic salen de las mejores universidades y aplican a diario ciencias
y tecnologías aprendidas ahí, las que, sin embargo, crean y reproducen el mal
desarrollo en el que trata de sobrevivir la mayor parte de la humanidad.

Estos planteamientos llevan a la idea de que hoy en día ya no es suficiente formar a los mejores profesionales en el aspecto académico y cumplir con las expectativas sobre su inserción laboral, sino que el reto es mucho mayor y consiste en formar a estudiantes que sean capaces de contribuir a la construcción de una sociedad más justa.

Para lograr lo antes mencionado, es deseable que los responsables de la Educación Superior se den a la tarea de identificar y trabajar a lo largo de la trayectoria universitaria, de manera deliberada, aquellas características vinculadas a la RSU que deberían estar presentes en los estudiantes universitarios al momento de egresar, que les permitan participar en procesos de transformación social.

Una vez que la Universidad ha definido aquellas características vinculadas a la RSU, existen instrumentos de medición que facilitan a las IES los procesos de reflexión relacionados con la formación de estudiantes socialmente responsables. Por ejemplo, el Modelo Universidad Construye País propone un instrumento dirigido a estudiantes, maestros y administrativos que permite conocer desde la perspectiva de cada actor, si la universidad está encaminando sus esfuerzos a formar en el ejercicio de sus funciones a estudiantes socialmente responsables (Fernández, Delpiano y De Ferari, 2006; Jiménez, 2008). 
Otro ejemplo de instrumento de medición lo propone National Clearinghouse for Leardership Programs (NCLP, 2015), el cual se basa en el Modelo para el cambio social (HERI, 1996; Komives et al., 2017) y autoevalúa si el estudiante reconoce poseer las características de un líder socialmente responsable.

Por otra parte, el Modelo SPRING propone un formato de evaluación de la responsabilidad pro-social con una rúbrica de autoevaluación del desempeño profesional que contempla criterios de evaluación y ejemplos de conductas socialmente responsables.

Existen otros instrumentos menos detallados que, sin surgir desde los modelos de formación, se han diseñado en el marco de la RSU y evalúan comportamientos y actitudes socialmente responsables en estudiantes universitarios, tales como el Cuestionario de Autoatribución de Comportamientos Socialmente Responsables (Davidovich, Espina y Navarro, 2005) y la Escala de medida del grado de Responsabilidad Social del universitario (Calle et al., 2008).

En la búsqueda hecha hasta el momento, en esta investigación de doctorado no se ha encontrado un instrumento de autoreporte en el contexto latinoamericano en el que el estudiante sea capaz de reconocer si posee o no las características vinculadas a la RSU. Por lo tanto, el objetivo del presente artículo es dar a conocer los resultados del proceso de construcción y validación de un instrumento para la autoevaluación de las características vinculadas a la Responsabilidad Social Universitaria (RSU) presentes en estudiantes.

\section{Revisión de la literatura}

Las características de un estudiante socialmente responsable han sido planteadas por diversos autores, clásicos o contemporáneos, con propuestas individuales o bien, otros autores que las condensan en modelos de formación para el cambio social en el marco de la Educación Superior. En el cuadro 1 se muestran algunas características deseables a encontrar en los agentes que promueven el cambio social, las cuales se presentan en el cuadro 1 .

Cuadro 1. Autores que han escrito sobre cambio social y características vinculadas a la RSU

\begin{tabular}{|c|c|}
\hline AUTORES & CARACTERÍSTICAS VINCULADAS A LA RSU \\
\hline $\begin{array}{l}\text { Benedicto (2016); Benedicto y } \\
\text { Morán (2002) }\end{array}$ & Ciudadanía \\
\hline Cruz y Sasia (2008) & Compromiso, Justicia social, Razonamiento ético \\
\hline Freire $(1998,2001)$ & $\begin{array}{l}\text { Autonomía, Cambio, Ciudadanía, Compromiso, } \\
\text { Conciencia, Congruencia, Disconformidad con las } \\
\text { injusticias, Esperanza, Indignación, Lectura de mundo, } \\
\text { Libertad, Razonamiento ético, Respeto a la diversidad }\end{array}$ \\
\hline Murillo y Hernández-Castilla (2014) & Justicia social \\
\hline Tedesco (2012) & Justicia social \\
\hline Vallaeys (s.f.) & $\begin{array}{l}\text { Ciudadanía, Compromiso, Conocimiento del contexto } \\
\text { para la solución de problemas, Desarrollo sostenible, } \\
\text { Empatía, Ética del diálogo, Justicia social, Solidaridad }\end{array}$ \\
\hline
\end{tabular}

Fuente: Elaboración propia.

Por otro lado, existen autores que, al considerar las características de la Responsabilidad Social con los fundamentos requeridos, formaron modelos que explican las variables y sus relaciones, incluyendo dimensiones de formación. 
Los dos primeros son modelos que surgen en el contexto de la RSU y tienen como objetivo impulsar la transformación social conjuntamente con actores de interés, desde una visión de desarrollo sostenible y justicia social. El tercer modelo, es una propuesta diseñada en Estados Unidos en el marco de la Educación Superior, que sin surgir en el marco de la RSU, tiene en común con los modelos previos la búsqueda de la transformación social a través de proponer una serie de características de los líderes socialmente responsables.

Cuadro 2. Modelos de formación para el cambio social

\begin{tabular}{|c|c|c|c|}
\hline $\begin{array}{l}\text { NOMBRE DEL } \\
\text { MODELO }\end{array}$ & AuTORES & DIMENSIONES & $\begin{array}{l}\text { CARACTERÍSTICAS VINCULADAS A LA } \\
\text { RSU }\end{array}$ \\
\hline \multirow{3}{*}{$\begin{array}{l}\text { Universidad } \\
\text { Construye País }\end{array}$} & \multirow{3}{*}{$\begin{array}{l}\text { Jiménez } \\
\text { (2008) }\end{array}$} & Personal & $\begin{array}{l}\text { Dignidad de la persona, Libertad, } \\
\text { Integridad }\end{array}$ \\
\hline & & Social & $\begin{array}{l}\text { Bien común y equidad social, } \\
\text { Desarrollo sostenible y medio } \\
\text { ambiente, Sociabilidad y solidaridad } \\
\text { para la convivencia, Aceptación y } \\
\text { aprecio por la diversidad, Ciudadanía, } \\
\text { democracia y participación }\end{array}$ \\
\hline & & Universitario & $\begin{array}{l}\text { Compromiso con la verdad, } \\
\text { Excelencia, Interdependencia y } \\
\text { transdisciplinariedad }\end{array}$ \\
\hline \multirow{3}{*}{$\begin{array}{l}\text { Responsabilidad } \\
\text { social a través de } \\
\text { Intervenciones } \\
\text { Prosociales para } \\
\text { generar } \\
\text { oportunidades } \\
\text { equitativas } \\
\text { (SPRING) }\end{array}$} & \multirow{3}{*}{$\begin{array}{l}\text { Jiménez } \\
(2014)\end{array}$} & $\begin{array}{l}\text { Responsabilidad } \\
\text { individual }\end{array}$ & $\begin{array}{l}\text { Empatía con la realidad social, Empatía } \\
\text { con los aspectos éticos en el ejercicio } \\
\text { de su ciudadanía, Confianza y } \\
\text { autonomía para tomar decisiones, } \\
\text { Dignidad humana }\end{array}$ \\
\hline & & $\begin{array}{c}\text { Responsabilidad } \\
\text { intersubjetiva }\end{array}$ & $\begin{array}{l}\text { Juicio crítico de la sociedad } \\
\text { globalizada, de la región y de los } \\
\text { impactos económicos, sociales y } \\
\text { medioambientales } \\
\text { Gestión de iniciativas sociales desde la } \\
\text { disciplina para la justicia y el bien } \\
\text { común }\end{array}$ \\
\hline & & $\begin{array}{c}\text { Responsabilidad } \\
\text { prospectiva }\end{array}$ & $\begin{array}{l}\text { Liderazgo prosocial } \\
\text { Gestión y actuación en la realidad, } \\
\text { Generación de un clima social } \\
\text { positivo }\end{array}$ \\
\hline \multirow{3}{*}{$\begin{array}{l}\text { Cambio social y } \\
\text { desarrollo del } \\
\text { liderazgo }\end{array}$} & \multirow{3}{*}{$\begin{array}{l}\text { HERI } \\
(1996) \\
\text { Komives y } \\
\text { otros } \\
(2017)\end{array}$} & Individual & $\begin{array}{cccc}\text { Conciencia } & \text { de uno } & \text { mismo, } \\
\text { Congruencia, } & \text { Compromiso } & \end{array}$ \\
\hline & & Grupal & $\begin{array}{l}\text { Colaboración, Propósito común, } \\
\text { Controversia con civilidad }\end{array}$ \\
\hline & & Comunitaria & Ciudadanía, Cambio \\
\hline
\end{tabular}

Fuente: Elaboración propia.

Además de los Modelos antes mencionados, existe un cuarto que, sin haber sido diseñado en el marco de la Educación Superior, es de amplio espectro, ya que se pensó para propiciar un cambio transformativo de manera colectiva en instituciones formales, no formales, así como también en comunidades con miras a la construcción de realidades más justas y equitativas.

El Modelo de Dimensiones de Cambio Social propuesto por Retolaza (2010) propone cuatro dimensiones para lograr un proceso de cambio transformativo: transformación personal, transformación de relaciones, transformación de patrones colectivos de pensamiento y acción y transformación de estructuras. Analizar el cambio desde estas 
cuatro dimensiones le permitirán a las instituciones plantear acciones más integrales y colaborativas (figura 1).

DIMENSIONES DE CAMBIO

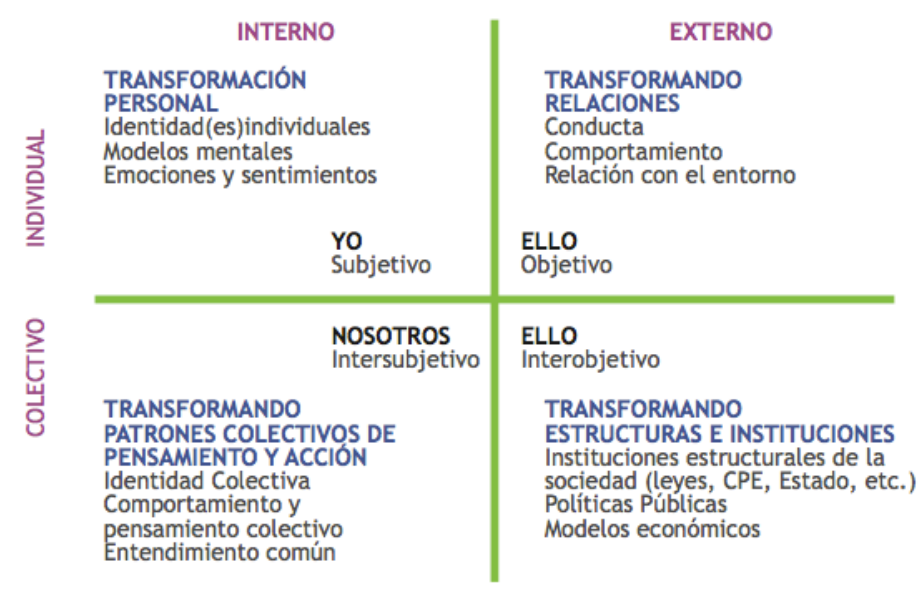

Figura 1. Modelo de dimensiones de cambio social de Retolaza (2010)

Fuente: Recuperado de Retolaza (2010, p. 7).

Cualquiera de las características vinculadas a la RSU se puede analizar desde los cuatro cuadrantes. Por ejemplo, la ciudadanía vista desde el cuadrante 1, implicaría que el estudiante se conciba así mismo como un buen ciudadano. Un estudiante que está en el cuadrante dos es un buen ciudadano en la práctica (comportamiento). Este tipo de ciudadanía propicia que el estudiante viva su condición de ciudadano como agente de cambio social, utilizando el diálogo y la empatía para transformar la relaciones con los otros y con su entorno. Una persona que se ubica en el cuadrante tres es capaz de debatir como ciudadano asuntos de preocupación de la colectividad y lograr un entendimiento común o pensamiento colectivo que apunte a la transformación social. Por último, un estudiante que se ubica en el cuadrante cuatro, debate asuntos de preocupación de la colectividad, diseñando proyectos, presentándolos en espacios tales como foros, y participando en su implementación (Benedicto y Morán, 2002; Retolaza, 2010).

\section{Método}

El obtener un instrumento que pudiera asociar las características de la Responsabilidad Social Universitaria con las propias del estudiante se hizo en cinco etapas: el trabajo de diseño y construcción estuvo conformado por tres, mientras que el de pilotaje, validación e instrumento final por dos. Se explica cada una de las etapas.

\section{Primera etapa: Revisión de la literatura}

De esta revisión resultó la lista de las características de un alumno socialmente responsable propuestas desde los Modelos que forman a estudiantes socialmente responsables en el marco de la Educación Superior y los autores que han escrito sobre cambio social. 
Las siete características que resultaron seleccionadas, son las que coinciden con la mayoría de los modelos y autores que han escrito sobre cambio social y además cuentan con un vasto sustento teórico. Las características vinculadas a la RSU que se utilizaron para construir el instrumento se presentan en el cuadro 3.

Cuadro 3. Características vinculadas a la RSU seleccionadas de los autores y modelos de cambio social

\begin{tabular}{|c|c|}
\hline CARACTERÍSTICA & DEFINICIÓN \\
\hline Conciencia & $\begin{array}{l}\text { Es el reconocimiento de los talentos, intereses, aspiraciones, valores, } \\
\text { creencias, preocupaciones, autoconcepto, motivaciones, limitaciones y } \\
\text { sueños personales con miras a ponerlos al servicio del otro y en la } \\
\text { construcción de una sociedad más justa (HERI, 1996; Komives et al., 2017, } \\
\text { Freire, 1998, 2001). Esta toma de conciencia personal fortalece en el } \\
\text { estudiante su confianza, identidad y autonomía para tomar decisiones y } \\
\text { comunicarlas con respeto y empatía con los otros (Komives et al., 2017; } \\
\text { Gamboa et al., 2014). } \\
\text { También implica reconocer con empatía la realidad social (Gamboa et al., } \\
\text { 2014) y estar informado para ser capaz de utilizar sus saberes especializados } \\
\text { en la solución de problemas de la sociedad (Vallaeys, s.f.). Este tipo de } \\
\text { conciencia Freire (1998) la llama "lectura del mundo". Incluye de igual } \\
\text { forma, la capacidad de comprender la lectura del mundo que los grupos } \\
\text { hacen de su contexto, valorando sus saberes y experiencias (Freire, 2001, } \\
\text { p. 94). Es una especie de conciencia social. También comprende una } \\
\text { conciencia ambiental, la cual consiste en satisfacer las necesidades presentes } \\
\text { sin comprometer los derechos de las generaciones futuras, es decir, contar } \\
\text { con una mentalidad para el desarrollo sostenible (Jiménez, 2008; Vallaeys, } \\
\text { 2013). Por último, es un estar en el mundo, con el mundo y con los otros y } \\
\text { preguntarse de manera permanente el significado de nuestra presencia y el } \\
\text { papel protagónico que se juega en la transformación social (Freire, 1998; } \\
\text { HERI, 1996; Komives et al., 2017; 2001). Es una conciencia trascendental. }\end{array}$ \\
\hline Compromiso & $\begin{array}{l}\text { Es comprometerse con la justicia social y el servicio a los demás desde las } \\
\text { necesidades de la propia comunidad (Cruz y Sasia, 2008; Freire, 2001; } \\
\text { Jiménez, 2008). Este compromiso durante el trayecto por la universidad } \\
\text { requiere del estudiante: pasión, credibilidad, responsabilidad, mantenerse } \\
\text { dedicado ante periodos de estrés, persistencia a pesar de los retos o desafíos, } \\
\text { cumplir los compromisos de manera oportuna y por arriba de las } \\
\text { expectativas, así como también, ser capaz de sostener profundos y } \\
\text { significativos compromisos (Komives et al., 2017). }\end{array}$ \\
\hline $\begin{array}{l}\text { Controversia con } \\
\text { civilidad }\end{array}$ & $\begin{array}{l}\text { Actuar con civilidad es comprender la naturaleza de un desacuerdo o } \\
\text { disputa y contar con la habilidad para continuar con el diálogo y el trabajo } \\
\text { colaborativo teniendo como propósito común la construcción de una } \\
\text { sociedad más justa y humana. También implica la capacidad de indignarse } \\
\text { ante las injusticias y atreverse a denunciarlas con sustento, mostrando en } \\
\text { todo momento respeto por los otros (Freire, 1998, 2001; HERI, 1996; } \\
\text { Komives et al., 2017). }\end{array}$ \\
\hline $\begin{array}{l}\text { Respeto a la } \\
\text { diversidad }\end{array}$ & $\begin{array}{l}\text { El respeto a la diversidad implica que el estudiante tome conciencia de la } \\
\text { interculturalidad y la multiculturalidad. La educación multicultural es la } \\
\text { coexistencia de grupos con culturas diferentes. El respeto a la diversidad } \\
\text { desde la multiculturalidad, implica reconocer la propia identidad cultural y } \\
\text { apreciar otras culturas. Por otra parte, la educación intercultural tiene } \\
\text { como propósito superar la coexistencia pasiva entre culturas, buscar la } \\
\text { manera de evolucionar en la convivencia y que dicha interacción sea } \\
\text { sostenible en sociedades multiculturales, de manera tal, que se viva un } \\
\text { ambiente de conocimiento mutuo, respeto y diálogo entre los diferentes } \\
\text { grupos culturales (Freire, 1998; Schmelkes, 2006; UNESCO, 2006). }\end{array}$ \\
\hline
\end{tabular}




\begin{tabular}{|c|c|}
\hline Ciudadanía & $\begin{array}{l}\text { Benedicto (2016) identifica dos tipos de ciudadanía juvenil: (i) aquella que } \\
\text { se centra en su condición o estatus y se alcanza al integrarse a la vida } \\
\text { laboral, cumpliendo una serie de deberes colectivos que el Estado regula. } \\
\text { Una ciudadanía que muestra señales de desconfianza hacia la vida pública y } \\
\text { se repliega hacia la privada; una ciudadanía más bien observadora del } \\
\text { manejo que otros hacen de los asuntos públicos y (ii) la ciudadanía como } \\
\text { agencia, aquella que no solo es estatus sino práctica a la vez. Este tipo de } \\
\text { ciudadanía permite al estudiante vivir su condición de ciudadano como } \\
\text { agente de cambio social, debatiendo asuntos de preocupación de la } \\
\text { colectividad, diseñando proyectos y presentándolos en espacios o } \\
\text { plataformas tales como foros y de ser posible, participando en su } \\
\text { implementación. Este tipo de ciudadanía es la que se desea formar en los } \\
\text { estudiantes universitarios (Benedicto, 2016; Benedicto y Morán, 2002). }\end{array}$ \\
\hline Justicia social & $\begin{array}{l}\text { La Universidad como institución formadora contribuye de forma decisiva a } \\
\text { la generación de una dinámica que trabaje desde y por la justicia social, o } \\
\text { bien, a reproducir o perpetuar las injusticias o desigualdades (Murillo y } \\
\text { Hernández-Castilla, 2014). De ahí la importancia de que los jóvenes } \\
\text { comprendan los retos de la justicia (pobreza, desigualdad, discriminación, } \\
\text { etc.) independientemente del entorno político, social, económico, ambiental } \\
\text { y mantengan una orientación que se incline preferentemente hacia los } \\
\text { ámbitos de mayor vulnerabilidad de su sociedad o a aquellos grupos } \\
\text { excluidos por la sociedad (Cruz y Sasia, 2008; Tedesco, 2012; Murillo y } \\
\text { Hernández-Castilla, 2014) y se cuestionen si su rol como profesionales es } \\
\text { adaptarse a la sociedad tal y como está o bien consiste en contribuir con su } \\
\text { profesión a la búsqueda permanente de una sociedad más justa y humana } \\
\text { (Freire, 2001; Tedesco, 2012; Murillo y Hernández-Castilla, 2014). }\end{array}$ \\
\hline Cambio & $\begin{array}{l}\text { Es creer que, aunque el cambio es difícil, es posible lograr un mundo más } \\
\text { humano y justo, y para lograrlo es necesario creer fielmente en la } \\
\text { posibilidad y fomentar la autenticidad de este sueño, es decir, hacer a un } \\
\text { lado ideologías fatalistas que estimulan la inmovilidad o la acomodación a } \\
\text { dichas realidades. En otras palabras, no quedarse en zona de confort, sino } \\
\text { estar abiertos a lo nuevo, a lo diferente, a la duda, a nuevas posibilidades de } \\
\text { construir un mundo mejor para uno mismo, para los demás; un mundo más } \\
\text { justo (Freire, 2001; HERI, 1996; Komives et al., 2017). }\end{array}$ \\
\hline
\end{tabular}

Fuente: Elaboración propia.

\section{Segunda etapa: Validez de contenido}

Se diseñó una primera versión del instrumento con 80 reactivos para señalar el nivel de acuerdo o desacuerdo utilizando una escala tipo Likert (a. muy de acuerdo, b) de acuerdo, c) en desacuerdo y d) muy en desacuerdo). Adicional a esta escala, al instrumento se le adaptó una siguiente columna para respuestas de "no sé” cuando no se tuvieran suficientes elementos para posicionarse ante el argumento.

El instrumento se sometió a un jueceo por 8 expertos. Para evaluar cada uno de los reactivos, se le pidió a los jueces contemplar los siguientes dos aspectos: (a) Claridad: hace referencia a si el planteamiento es de fácil comprensión o de lo contrario, es confuso. Si el reactivo es confuso, se le solicita al juez escribir la propuesta de redacción; (b) Pertinencia: se refiere a la correspondencia entre el reactivo y la característica; (c) Permanencia: hace referencia a si el reactivo debe permanecer como parte del instrumento porque responde a los objetivos del estudio.

De los ochenta reactivos sometidos a los jueces, 40 cumplieron con la validez de contenido y fueron aprobados. Estos reactivos estaban agrupados en función de las siete características de estudiantes socialmente responsables seleccionadas en la primera etapa de la construcción del instrumento (cuadro 4). 
Cuadro 4. Reactivos del instrumento que cumplieron con la validez de contenido

\section{CONCIENCIA}

Soy capaz de identificar aspectos de mi persona que puedo poner al servicio de los demás.

Estoy conciente de la importancia del autocuidado de mi persona como una manera de ser socialmente responsable.

Me interesa comprender el sentido de mi presencia en este mundo.

Hago un ejercicio permanente de auto observación que me permite darme cuenta cuando mi pensamiento. no reconoce los derechos y necesidades de los demás.

Soy capaz de reconocer mis limitaciones.

Me considero una persona digna de confianza.

Puedo aprovechar la retroalimentación que recibo de mi actuar, aun cuando sea negativa.

Entiendo que ser parte de este mundo implica una responsabilidad ética por el entorno.

Me preocupo por mantenerme informado acerca de las problemáticas que afectan a mi País.

Conozco las problemáticas locales y su relación con factores nacionales y mundiales.

Durante la realización de mis actividades cotidianas algo un alto para reflexionar sobre los pensamientos y experiencias que me afectan y afectan a los demás.

Me considero capaz de analizar los problemas de mi comunidad de manera crítica.

\section{Compromiso}

Me apasiona trabajar por causas que considero justas.

No me doy por vencido ante retos universitarios complejos.

Por lo general, no acepto más compromisos de los que puedo sobrellevar.

Me motivo y motivo a otros a permanecer dedicado a los compromisos universitarios.

Completo los proyectos o compromisos universitarios por arriba de las expectativas de mis profesores.

\section{CONTROVERSIA CON CIVILIDAD}

Me siento cómodo siendo transparente con los demás acerca de mis intenciones, valores y prioridades.

Me invito e invito a los demás a comprender y dialogar nuestras diferencias.

Soy capaz de manifestarme contra situaciones que desde mi perspectiva son injustas aun cuando exista controversia a mi alrededor.

Cuando un estudiante no se compromete con el trabajo grupal, se lo expreso a través de una conversación constructiva.

Se me facilita mediar conflictos dentro del trabajo colaborativo en los espacios universitarios.

Me atrevo públicamente a disentir con sustento del punto de vista colectivo cuando no se respeta la dignidad humana.

\section{RESPETO A LA DIVERSIDAD}

Estoy abierto a trabajar con grupos diversos (raza, género, cultura, religión, orientación sexual).

Estoy convencido que trabajar con personas diversas es una herramienta poderosa para encontrar soluciones innovadoras a los problemas.

En el trabajo con grupos diversos procuro que todos tengamos oportunidad de expresar nuestras ideas.

Procuro, al trabajar con grupos diversos, crear una atmósfera de confianza y respeto.

Como profesionista me gustaría hacer lo posible para vivir en un mundo donde se respete y aprecie la diversidad humana (raza, nacionalidad, cultura).

Reconozco mi propia identidad cultural y aprecio otras culturas.

El estudio de mi profesión me ha brindado herramientas para sensibilizar a los demás sobre la importancia de respetar al otro en su diversidad (racismo, discriminación, preferencias sexuales, igualdad de género).

\section{CIUDADANía}

Conozco sobre el ejercicio de los derechos humanos y las responsabilidades asociadas.

Aprovecho las oportunidades que se me presentan para comprometerme con la realización de un mundo más justo.

\section{JUSTICIA SOCIAL}

Como estudiante me siento con la confianza para hacer algo ante situaciones de injusticia.

Estoy convencido que el estilo de vida sustentado en la justicia, equidad y dignidad es el adecuado.

El estudio de mi profesión me ha permitido percatarme de la existencia de exclusión e inequidad en los grupos en los que me desenvuelvo (salón de clases, Universidad, vecindario). 
En mi acción profesional, tomo en cuenta a las personas y grupos que están poco representados y marginados.

\section{CAMBIO}

Tengo la certeza del que el cambio social es difícil pero posible.

Estoy conciente que estoy en el mundo para contribuir de modo responsable a su

transformación social.

Siento que nada se puede hacer ante la falta de responsabilidad global.

Ante los problemas de mi País, prefiero tomar una postura optimista antes que una postura fatalista.

Fuente: Elaboración propia

\section{Tercera etapa: rediseño del instrumento}

Con base en los resultados obtenidos del jueceo, se buscó un modelo teórico que integrara los reactivos que cumplieron con la validez de contenido de la etapa dos. En esta búsqueda se encontró el Modelo de Dimensiones de Cambio Social de Retolaza (2010), el cual propone cuatro dimensiones para lograr el cambio social: transformación personal, transformación de relaciones, transformación de patrones colectivos y transformación de estructuras y organizaciones. En función de estas dimensiones se rediseñó el instrumento quedando un total de 60 reactivos, de los cuales 40 fueron seleccionados en el jueceo previo. Los 20 reactivos nuevos fueron diseñados con el fin de que el instrumento refleje la riqueza conceptual del modelo. En el cuadro 5, se presentan los nuevos reactivos relacionados con las dimensiones del Modelo. Cabe mencionar que estos reactivos fueron sometidos al juicio de tres expertos.

Cuadro 5. Reactivos que se adicionan al instrumento para alinearlo a las Dimensiones de Cambio Social de Retolaza (2010)

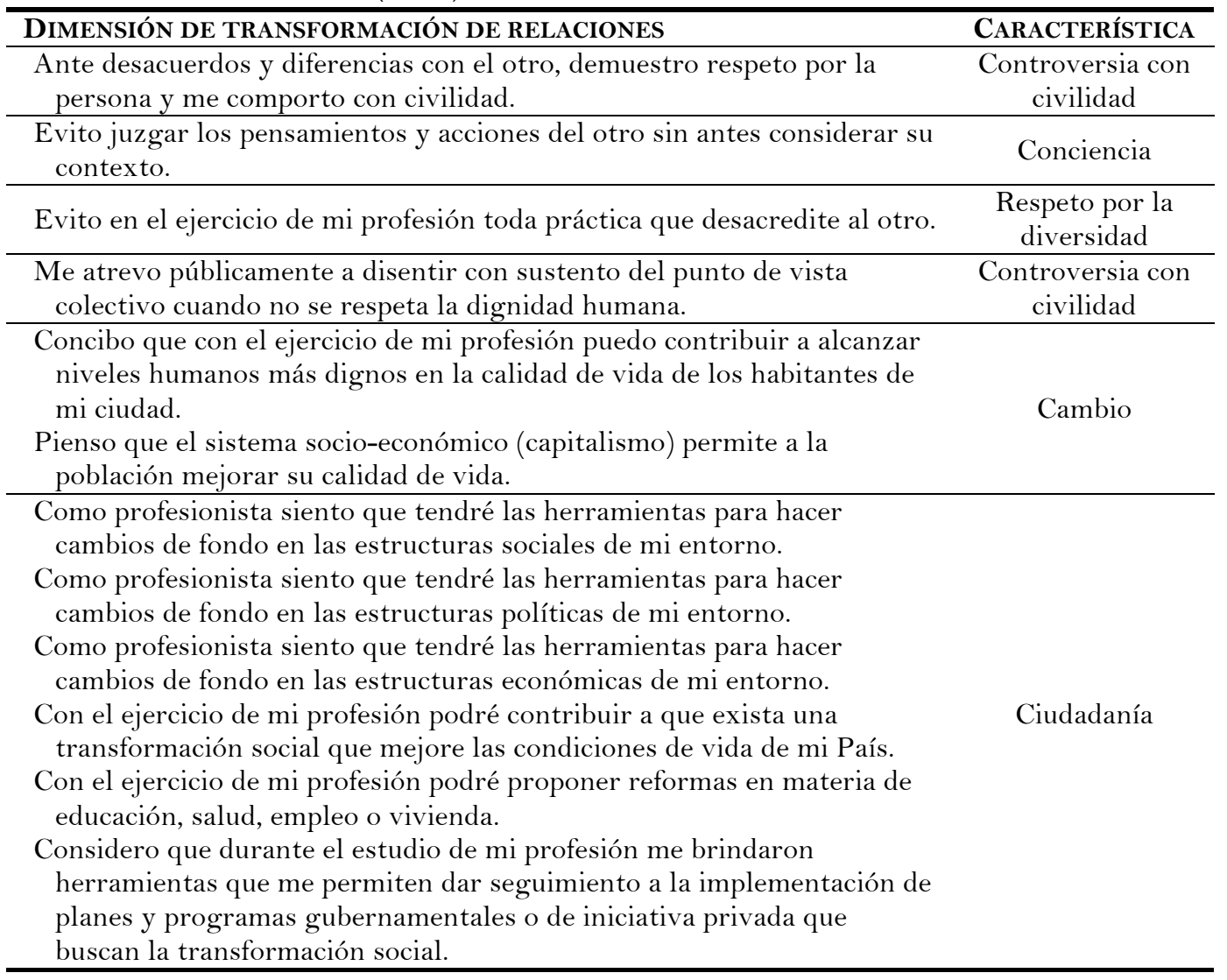


Como profesional me visualizo trabajando en el sector público para desde

ahí mejorar las prácticas que contribuyan a la transformación social.

Considero que con el ejercicio de mi profesión podré contribuir a la reducción de la pobreza de mi Estado.

Me interesa utilizar mi profesión como un medio para contribuir a la reducción de la pobreza de mi Estado.

Considero que la corrupción es un problema de urgente solución y desde ya estoy tomando acciones para combatirla.

Considero que en mi Estado no hay igualdad de oportunidades y cuando ejerza mi profesión será una de las metas que perseguiré.

Como futuro profesional procuraré no aceptar trabajos o contratar a Justicia social personas por una relación de compadrazgo y padrinazgo.

Como profesional me gustaría incidir en el diseño, implementación y evaluación de políticas públicas que mejoren la calidad de vida de los grupos minoritarios (raza, etnia, preferencia sexual).

Como profesional me gustaría incidir en el diseño, implementación y evaluación de políticas públicas que mejoren la calidad de vida de grupos vulnerables (niños, mujeres, personas de la tercera edad, etc.).

Fuente: Elaboración propia

En el cuadro 6 se muestra la distribución por reactivo para la versión 2 del instrumento.

Cuadro 6. Distribución de reactivos por dimensión

\begin{tabular}{lc}
\hline \multicolumn{1}{c}{ DIMENSIÓN } & No DE REACTIVOS $^{\circ}$ \\
\hline Transformación personal & 19 \\
Transformación de relaciones & 14 \\
Transformación de patrones colectivos de pensamiento y acción & 12 \\
Transformación de estructuras & 15 \\
\hline Total de reactivos & 60 \\
\hline
\end{tabular}

Fuente: Elaboración propia.

\section{Cuarta etapa: Pilotaje del instrumento}

Se piloteó el instrumento con una muestra de 102 estudiantes. La muestra con la que se trabajó fueron estudiantes de licenciatura de dos universidades privadas ubicadas en el centro y sur de México que pertenecen a la Federación de Instituciones Mexicanas particulares de Educación Superior (FIMPES), asociación que acredita la calidad de las instituciones mexicanas particulares con el propósito de que puedan servir mejor a la nación. El motivo por el cual se decide trabajar con universidades privadas es por la escasa investigación existente en éstas en comparación con la disponible de universidades públicas con relación al tema de RSU (Pérez y Vallaeys, 2016).

Los criterios que se consideraron para seleccionar a las universidades privadas que participaron fueron:

- Declarar en su filosofía institucional la postura y el compromiso que tienen con la sociedad.

- Contemplar de manera curricular o no curricular experiencias universitarias que formen a estudiantes socialmente responsables.

- Estar acreditadas en calidad por la Federación de Instituciones Particulares de Educación Superior (FIMPES) y haber obtenido el dictamen de Lisa y llana en su última acreditación, lo cual significa el máximo nivel de calidad que otorga dicha institución. 
Una vez seleccionadas las universidades, se les pidió a los directores de asuntos estudiantiles los datos sobre población total y por programa académico según género para determinar el número de alumnos a los cuales aplicar la prueba piloto. El tipo de muestreo fue no probabilístico, ya que la participación de los estudiantes no fue decidida por el investigador sino por la propia universidad. En total participaron 102 estudiantes del semestre enero-julio 2018 de los programas de licenciatura que ambas universidades tenían en común, a saber: Diseño Gráfico, Contaduría, Ingeniería Industrial, Mercadotecnia y Psicología.

Quinta etapa: Validez de constructo

Utilizando el programa SPSS se realizó un análisis estadístico de consistencia interna con la prueba alfa de Cronbach para determinar la fiabilidad del instrumento. La primera corrida de datos arrojó un alpha de cronbach de 0,928 con 60 reactivos. Adicionalmente, se realizó un análisis por reactivo con el fin de comprobar aquellos ítems que discriminaban, encontrando 8 reactivos que tenían baja consistencia interna. Una vez eliminados, se volvió a correr la prueba alfa obteniendo un alfa de 0,936. Estos 52 reactivos fueron revisados por dos jueces porque 5 reactivos parecían ser ajenos al constructo donde quedaron ubicados estadísticamente. Se acordó eliminarlos quedando un total de 47 reactivos para los que se corrió nuevamente la prueba de alpha que obtuvo un puntaje de 0,932 .

También se realizó el análisis factorial a través de la prueba de componentes principales con rotación Varimax para determinar y validar las dimensiones, y asimismo obtener la varianza explicada del conjunto de reactivos. Este análisis arrojó cuatro dimensiones (cuadro 7) que corresponden a las propuestas por el Modelo de Dimensiones de Cambio Social de Retolaza.

Cuadro 7. Distribución de reactivos por dimensiones y su valor Alpha

\begin{tabular}{lcc}
\hline \multicolumn{1}{c}{ DIMENSIÓN } & ALFA & NO. DE REACTIVOS \\
\hline Transformación personal & 0,804 & 9 \\
Transformación de relaciones & 0,811 & 12 \\
Transformación de patrones colectivos & 0,815 & 11 \\
Transformación de estructura e instituciones & 0,875 & 15 \\
\hline Total de reactivos & & 47 \\
\hline
\end{tabular}

Fuente: Elaboración propia

Finalmente, se muestra cómo quedaron distribuidas las características de la RSU presentes en estudiantes universitarios en función de las cuatro dimensiones del Modelo de Retolaza. Los reactivos se distribuyen en los cuadros 8, 9, 10 y 11.

Cuadro 8. Dimensión transformación personal

\begin{tabular}{lcc}
\hline REACTIVo & PESO FACTORIAL & CARACTERístICA \\
\hline $\begin{array}{l}\text { 1. Soy capaz de identificar aspectos de mi persona } \\
\text { que puedo poner al servicio de los demás. }\end{array}$ & 0,574 & Conciencia \\
$\begin{array}{l}\text { 2. Estoy conciente de la importancia del } \\
\text { autocuidado de mi persona como una manera de }\end{array}$ & 0,600 & Conciencia \\
$\quad \begin{array}{l}\text { ser socialmente responsable. } \\
\text { 3. Me interesa comprender el sentido de mi } \\
\text { presencia en este mundo. }\end{array}$ & 0,648 & Conciencia \\
$\begin{array}{l}\text { 4. Soy una persona que alza la voz cuando no se } \\
\text { respeta la dignidad humana. }\end{array}$ & 0,626 & $\begin{array}{c}\text { Controversia con } \\
\text { civilidad }\end{array}$ \\
$\begin{array}{l}\text { 5. Me apasiona trabajar por causas que considero } \\
\text { justas. }\end{array}$ & 0,659 & Compromiso
\end{tabular}


6. Como estudiante me siento con la confianza para hacer algo ante situaciones de injusticia.

0,674

0,551

0,665

0,624

Conciencia observación que me permite darme cuenta cuando mi pensamiento no reconoce los derechos y necesidades de los demás.

Fuente: Elaboración propia

Cuadro 9. Dimensión transformación de relaciones

\begin{tabular}{|c|c|c|}
\hline REACTIVO & PESO FACTORIAL & CARACTERÍSTICA \\
\hline $\begin{array}{l}\text { 1. Puedo aprovechar la retroalimentación que recibo } \\
\text { de mi actuar, aun cuando sea negativa. }\end{array}$ & 0,546 & Conciencia \\
\hline $\begin{array}{l}\text { 2. Entiendo que ser parte de este mundo implica una } \\
\text { responsabilidad ética por el entorno. }\end{array}$ & 0,626 & Conciencia \\
\hline $\begin{array}{l}\text { 3. Aprovecho las oportunidades que se me presentan } \\
\text { para comprometerme con la realización de un } \\
\text { mundo más justo. }\end{array}$ & 0,534 & Ciudadanía \\
\hline $\begin{array}{l}\text { 4. Me invito e invito a los demás a comprender y } \\
\text { dialogar nuestras diferencias. }\end{array}$ & 0,608 & $\begin{array}{l}\text { Controversia con } \\
\text { civilidad }\end{array}$ \\
\hline $\begin{array}{l}\text { 5. Me preocupo por mantenerme informado acerca } \\
\text { de las problemáticas que afectan a mi País. }\end{array}$ & 0,673 & Conciencia \\
\hline $\begin{array}{l}\text { 6. Ante los problemas de mi País, prefiero tomar una } \\
\text { postura optimista antes que una postura fatalista. }\end{array}$ & 0,566 & Cambio \\
\hline $\begin{array}{l}\text { 7. Conozco las problemáticas locales y su relación } \\
\text { con factores nacionales y mundiales. }\end{array}$ & 0,838 & Conciencia \\
\hline $\begin{array}{l}\text { 8. Ante desacuerdos y diferencias con el otro, } \\
\text { demuestro respeto por la persona y me comporto } \\
\text { con civilidad. }\end{array}$ & 0,606 & $\begin{array}{l}\text { Controversia con } \\
\text { civilidad }\end{array}$ \\
\hline $\begin{array}{l}\text { 9. Me motivo y motivo a otros a permanecer } \\
\text { dedicado a los compromisos universitarios. }\end{array}$ & 0,713 & Compromiso \\
\hline $\begin{array}{l}\text { 10. Evito juzgar los pensamientos y acciones del } \\
\text { otro sin antes considerar su contexto. }\end{array}$ & 0,731 & Conciencia \\
\hline $\begin{array}{l}\text { 11. Evito en el ejercicio de mi profesión toda } \\
\text { práctica que desacredite al otro. }\end{array}$ & 0,699 & $\begin{array}{l}\text { Respeto por la } \\
\text { diversidad }\end{array}$ \\
\hline $\begin{array}{l}\text { 12. Completo los proyectos o compromisos } \\
\text { universitarios por arriba de las expectativas de mis } \\
\text { profesores. }\end{array}$ & 0,738 & Compromiso \\
\hline
\end{tabular}

Fuente: Elaboración propia.

Cuadro 10. Dimensión de patrones culturales

\section{REACTIVO}

1. El estudio de mi profesión me ha permitido percatarme de la existencia de exclusión e inequidad en los grupos en los que me desenvuelvo (salón de clases, Universidad, vecindario).

2. El estudio de mi profesión me ha brindado herramientas para sensibilizar a los demás sobre la importancia de respetar al otro en su diversidad (racismo, discriminación, preferencias sexuales, igualdad de género).

3. Estoy abierto a trabajar con grupos diversos (raza, género, cultura, religión, orientación sexual).
Peso FaCtorial CARACTERística

$0,746 \quad$ Justicia social
diversidad diversidad 
4. Estoy convencido que trabajar con personas diversas es una herramienta poderosa para encontrar soluciones innovadoras a los problemas. diversidad

5. En el trabajo con grupos diversos procuro que todos tengamos oportunidad de expresar nuestras ideas.

Respeto a la

6. Procuro, al trabajar con grupos diversos, crear una atmósfera de confianza y respeto.

Respeto a la

7. Como profesionista me gustaría hacer lo posible para vivir en un mundo donde se respete y aprecie la diversidad humana (raza, nacionalidad, cultura).

8. En mi acción profesional, tomo en cuenta a las personas y grupos que están poco representados y marginados.

9. Me atrevo públicamente a disentir con sustento del punto de vista colectivo cuando no se respeta la dignidad humana.

10. Reconozco mi propia identidad cultural y aprecio otras culturas.

11. Concibo que con el ejercicio de mi profesión puedo contribuir a alcanzar niveles humanos más dignos en la calidad de vida de los habitantes de mi ciudad.

1. Como profesionista siento que tendré las herramientas para hacer cambios de fondo en las

2. Como profesionista siento que tendré las herramientas para hacer cambios de fondo en las estructuras políticas de mi entorno.

3. Como profesionista siento que tendré las herramientas para hacer cambios de fondo en las estructuras económicas de mi entorno.

4. Con el ejercicio de mi profesión podré contribuir a que exista una transformación social que mejore las condiciones de vida de mi País.

5. Con el ejercicio de mi profesión podré proponer reformas en materia de educación, salud, empleo o vivienda.

6. Considero que la corrupción es un problema de urgente solución y desde ya estoy tomando acciones para combatirla.

7. Considero que en mi Estado no hay igualdad de oportunidades y cuando ejerza mi profesión será una de las metas que perseguiré.

8. Como futuro profesional procuraré no aceptar trabajos o contratar a personas por una relación de compadrazgo y padrinazgo.

9. Como profesional me gustaría incidir en el diseño, implementación y evaluación de políticas públicas que mejoren la calidad de vida de los grupos minoritarios (raza, etnia, preferencia sexual).

10. Como profesional me gustaría incidir en el diseño, implementación y evaluación de políticas públicas que mejoren la calidad de vida de 
grupos vulnerables (niños, mujeres, personas de

la tercera edad, etc.).

11. Considero que durante el estudio de mi profesión me brindaron herramientas que me permiten dar seguimiento a la implementación de planes y programas gubernamentales o de iniciativa privada que buscan la transformación social.

12. Como profesional me visualizo trabajando en el sector público para desde ahí mejorar las prácticas que contribuyan a la transformación social.

13. Considero que con el ejercicio de mi profesión podré contribuir a la reducción de la pobreza de mi Estado.

$\begin{array}{lc}0,442 & \text { Ciudadanía } \\ 0,861 & \text { Ciudadanía } \\ 0,606 & \text { Justicia social } \\ 0,471 & \text { Justicia social } \\ 0,764 & \text { Cambio }\end{array}$

14. Me interesa utilizar mi profesión como un medio para contribuir a la reducción de la pobreza de mi Estado.

15. Pienso que el sistema socio-económico (capitalismo) permite a la población mejorar su calidad de vida.

Fuente: Elaboración propia.

\section{Discusión y conclusiones}

La globalización ha evidenciado con mayor claridad desafíos mundiales tales como la pobreza, la desigualdad, la discriminación, la insostenibilidad del medio ambiente, entre otros, cuestionando la pertinencia social de las Universidades, entendida en palabras de Tünnermann (2013) como la capacidad de tomar en cuenta los desafíos de la sociedad en apego a su misión.

Para que las universidades estén a la altura de estos desafíos deben cuestionarse si es suficiente con formar a buenos profesionales y cumplir únicamente con las expectativas sobre su inserción laboral. Hoy en día, el reto es mucho mayor y consiste en trabajar para un futuro sostenible a través de la formación de estudiantes socialmente responsables que puedan contribuir con su profesión a la transformación de la sociedad. En otras palabras, frente a estos desafíos, la educación continúa siendo el instrumento para poder pensar en un mundo donde exista paz, libertad y justicia (Delors, 1996).

La Universidad, en su esfuerzo por contribuir a la transformación de la sociedad, está en la permanente búsqueda de mecanismos que le permitan mejorar las prácticas educativas que mejor le lleven a este objetivo. Algunos de los mecanismos que se han encontrado para encaminarse a dicha transformación son a través del diseño, implementación y evaluación de programas y políticas para la RSU.

En el marco de la evaluación de la RSU, existen diversas propuestas teóricas con relación a los modelos de formación para el cambio social, pero todas tienen un desarrollo incipiente en el diseño, construcción y validación de instrumentos que le permitan a las Instituciones de Educación Superior indagar cómo los estudiantes se perciben para la transformación social. El interés por conocer la perspectiva del joven universitario a través de un instrumento de autoreporte, es de vital importancia, ya que es a él a quien van dirigidos los impactos de la RSU. 
Este instrumento, que autoevalúa las características vinculadas a la RSU presentes en estudiantes universitarios, se relaciona con las cuatro dimensiones del Modelo de Cambio Social de Retolaza, lo que le da fortaleza teórica. Por otra parte, con base en los resultados de confiabilidad y validez encontradas en el diseño y validación del instrumento, se puede decir que posee alta consistencia interna general, ya que arrojó un alfa general o,932 y alta consistencia interna en cada una de las cuatro dimensiones del modelo: transformación personal $(0,804)$, transformación de relaciones $(0,811)$, transformación de patrones colectivos $(0,815)$ y transformación de estructuras e instituciones $(0,875)$.

El instrumento puede tener múltiples usos, entre los cuales están indagar sobre las características vinculadas a la RSU presentes en estudiantes universitarios desde distintas áreas de conocimiento, o bien, analizar si hay diferencia significativa en los estudiantes que ingresan a la universidad, los estudiantes que están a la mitad de su trayecto universitario, o estudiantes que están por egresar. Estos análisis se pueden reflexionar desde: 1) el puntaje global que arroja la escala sobre las características de la RSU; 2) el puntaje obtenido en cada una de las características vinculadas a la RSU; 3) las cuatro dimensiones para el cambio social que plantea el Modelo de Retolaza para lograr la transformación social.

Los resultados del instrumento pueden ser de utilidad para las universidades, ya que permiten analizar si los esfuerzos institucionales están impactando la mirada del estudiante para la transformación social. Por otra parte, el docente puede reflexionar, con los resultados de la aplicación del instrumento, qué tanto su práctica educativa impacta en la manera como el estudiante se concibe a sí mismo y concibe su profesión.

Por último, este instrumento de autoreporte le permite al estudiante reflexionar sobre la situación del contex to en el que vive, sobre el rol que juega como persona y profesional en la búsqueda del cambio social, de tal forma que, junto con otros mecanismos de reflexión, emerja un joven con deseos de transformar la realidad injusta en conjunto con otros y que sea capaz, como dice Freire (1972), de proyectar su esencia sobre ella, es decir, humanizarla (como se citó en Murillo y Hernández-Castilla, 2014).

\section{Referencias}

Benedicto, J. (2016). La ciudadanía juvenil: Un enfoque basado en las experiencias vitales de los jóvenes. Revista Latinoamericana de Ciencias Sociales, Niñez y Juventud, 14(2), 925-938. https://doi.org/10.11600/1692715x.14203210415

Benedicto, J. y Morán, M. (2002). La construcción de una ciudadanía activa entre los jóvenes. Madrid: Injuve.

Calle, C. de la, García, J. M., Giménez, P. y Ortega, M. (2008). Validación y medida de la responsabilidad social universitaria en la universidad. Revista Complutense de Educación, $19(2), 385-404$.

Cruz, C. de la (2011). La clave de la responsabilidad social de las universidades está en la respuesta no en la pregunta: ¿̇Al servicio de quién se pone el bien público que promueven? Recuperado de https://www.mineducacion.gov.co/cvn/1665/w3-article-261550.html

Cruz, C. y Sasia, P. (2008). La responsabilidad de la universidad en el proyecto de construcción de una sociedad. Revista de Educación Superior y Sociedad. Nueva Época, 13(2), 21-51. 
Davidovich, M., Espina, A., Navarro, G. y Salazar, L. (2005). Construcción y estudio piloto de un cuestionario para evaluar comportamientos socialmente responsables en estudiantes universitarios. Revista de Psicología, 15(1), 125-139.

Delors, J. (1996). La educación encierra un tesoro. Ciudad de México: Correo de la UNESCO.

Fernández, C., Delpiano, C. y De Ferari, J. M. (2006). Responsabilidad social universitaria. Una manera de ser universidad. Teoría y práctica de la experiencia chilena. Santiago de Chile: Proyecto Universidad Construye País.

Freire, P. (1998). Política y educación. Barcelona: Siglo XX1.

Freire, P. (2001). Pedagogía de la indignación. Madrid: Morata.

Gamboa, A., Jiménez, M., Jiménez, G. y Lombardo, P. (2014). Formación en responsabilidad prosocial: Caminos latinoamericanos de una innovación curricular. Valparaíso: Proyecto Europeo Alfa III SPRING.

HERI. (1996). A social change model of leadership development. College Park, MD: National Clearinghouse for Leadership Programs.

Jiménez, M. (2008). ¿Cómo medir la percepción de la responsabilidad social en los diversos estamentos de la universidad? Una experiencia concreta. Revista Educación Superior y Sociedad: Nueva Época, 13(2), 140-161.

Komives, S. (2017). Leadership for a better world. Understandign the social change model. Nueva York, NY: Jossey-Bass.

Murillo, F. J. y Hernández-Castilla R. (2014). Liderando escuelas justas para la justicia social. Revista Internacional para la Justicia Social, 3(2),13-32.

UNESCO. (2006). Directrices de la UNESCO sobre educación intercultural. Recuperado de http://unesdoc.unesco.org/images/O014/O01478/147878s.pdf

UNESCO. (2016). Educación para la ciudadanía. Preparar a los educandos para los retos del siglo XXI. París: UNESCO.

ONU. (2019). Objetivos de desarrollo sostenible. Recuperado de http://www.onu.org.mx/agenda2030/objetivos-del-desarrollo-sostenible/

Pérez, J. y Vallaeys, F. (2016). Prácticas y modelos de responsabilidad social universitaria en México: Proceso de transformación de la universidad. Ciudad de México: ANUIES.

Retolaza, I. (2010). Teoría del cambio. Un enfoque de pensamiento acción para navegar en la complejidad de los procesos de cambio social. Ámsterdam: PNUD.

Schmelkes, S. (2006). La interculturalidad en la educación básica. Recuperado de http://www.socolpe.org/data/normalarmenia/BIBLIOGRAFIA/interculturalidad_educa cion_basica_schmelkes.pdf

Tedesco, J. (2012). Educación y justicia social en América Latina. Buenos Aires: Fondo de Cultura Económica de Argentina.

Tünnermann, C. (2013). Diez respuestas de la educación superior a los desafíos contemporáneos. Universidades, 56, 5-14.

Vallaeys, F. (2013). Virtud, justicia, sostenibilidad: Una ética de tres dimensiones para la responsabilidad social de las organizaciones. Recuperado de http://blog.pucp.edu.pe/blog/wpcontent/uploads/sites/54/2014/11/arti_cirs_2013.pdf

Vallaeys, F. (2016). Introducción a la responsabilidad social universitaria RSU. Barranquilla: Ediciones Universidad Simón Bolívar. 
Vallaeys, F. (2018). Las diez falacias de la responsabilidad social universitaria. Revista Digital de Investigación en Docencia Universitaria, 12(1), 34-58. https://doi.org/10.19083/ridu.12.716

Vallaeys, F. (s.f.). ¿Qué es la responsabilidad social universitaria? Lima: Pontificia Universidad Católica del Perú.

\section{Breve CV de las autoras}

\section{Valentina Bolio Domínguez}

Profesor de tiempo completo de la Universidad Marista de Mérida. Estudió la Licenciatura en psicología (Universidad Marista de Mérida), la Maestría en Tecnologías de la Educación (Universidad de Salamanca, España) y la Maestría en Investigación Educativa (Universidad Autónoma de Yucatán). Actualmente es Directora de Calidad Educativa en la Universidad Marista de Mérida y coordina los esfuerzos de acreditación a nivel institucional y por programa. Es candidato a doctor. Ha publicado artículos en plataformas nacionales. ORCID ID: https://orcid.org/0000-0002-7758-2067. Email: vbolio@marista.edu.mx

\section{Leny Michele Pinzón Lizarraga}

Profesor investigador de tiempo completo en el Instituto Tecnológico de Mérida. Sus estudios comprenden Licenciatura en Ciencias de la Educación (Universidad de Monterrey), Maestría en Administración (I.T.E.S.M.), y Doctorado en Análisis Estratégico y Desarrollo Sustentable (Universidad del Mayab). Perfil Deseable PROMEP. Líder del CA: Gestión de pequeñas y medianas empresas. Presidenta del Consejo de Posgrado de la Maestría en Gestión Administrativa. Imparte cátedra en las licenciaturas y maestrías del Instituto Tecnológico de Mérida. Forma parte del Colegio de Profesores del Doctorado en Educación de la Universidad Marista de Mérida. Ha publicado artículos en Revistas Nacionales e Internacionales, Libros y Capítulos de libros. ORCID ID: https://orcid.org/0000-0001-9108-5000. Email: lenypinzon@gmail.com 